\title{
A novel EGFR-targeted gene delivery system based on complexes self-assembled by EGF, DNA, and activated PAMAM dendrimers
}

This article was published in the following Dove Press journal:

International Journal of Nanomedicine

23 August 2012

Number of times this article has been viewed

Zhe Yin*

Nan Liu*

Mingshu Ma

Lan Wang

Yanli Hao

Xiaoning Zhang

Laboratory of Pharmaceutics, School of Medicine, Tsinghua

University, Beijing, China

*These authors contributed equally to this work
Correspondence: Xiaoning Zhang

School of Medicine, Tsinghua University,

Beijing 100084, China

Tel +861062785489

Fax +86106278 5410

Email drugman@mail.tsinghua.edu.cn

Yanli Hao

School of Medicine, Tsinghua University,

Beijing 100084, China

$\mathrm{Tel}+861062785489$

Fax +86106278 5410

Email haoyanli@tsinghua.edu.cn
Abstract: Epidermal growth factor receptor (EGFR)-targeted gene delivery is a promising approach in gene therapy against EGFR-positive cancer. In addition, macromolecules, such as polyamidoamine (PAMAM) dendrimers, are potential nonviral gene carriers in this therapy because of their biocompatibility and modifiable features. To achieve the goal of selectively enhancing the transfection efficiency in EGFR-positive cancer cells, the researchers developed chemical approaches of EGF-dendrimer conjugate, which were effective but complicated. Studies on liposomes reveal that self-assembly is another effective but simpler approach in EGF modification. Moreover, properly activated PAMAM dendrimers exhibit higher transfection efficiency, but little research has been done on its ligand-modification. In this study, we developed and characterized a novel gene-delivery system based on activated EGF-dendriplexes, which is formed via self-assembly by EGF and complexes prepared by activated PAMAM dendrimer and plasmid DNA. Such complexes exhibit desired features compared to nonmodified or nonactivated dendriplexes in vitro, including selective enhancement of transfection efficiency in EGFR-positive cells, decreased cytotoxicity, and low agonist effect. In vivo experimentation shows their EGFR-positive tumor targeted biodistribution and increased transfection efficiency at EGFR-positive tumors. Our results demonstrated that activated EGF-dendriplexes are safe and effective carriers for delivering gene drugs to EGFR-positive cells, which makes these complexes a promising targeted nonviral gene-delivery system for auxiliary cancer therapy.

Keywords: activated dendriplexes, transfection efficiency, EGFR targeting, self-assembled EGF modification

\section{Introduction}

In tumor gene therapy, it is an ideal strategy to deliver gene drugs selectively to tumor tissues by nonviral vectors. ${ }^{1}$ Such therapies can enhance the effectiveness and reduce the cytotoxicity of carriers to normal tissues and potential risks of overexpressing tumor-suppresser genes in normal tissues. ${ }^{2}$ A promising class of nonviral gene carrier candidates in drug-delivery systems is macromolecules. These molecules, used with DNA plasmids, can form nanosized complexes, whose enhanced permeation and retention effect (EPR) leads to passive targeting at tumor sites, ${ }^{3,4}$ and whose surface can be modified with targeting ligands to reach active targeting. ${ }^{1,5}$ Some essential features of macromolecular carriers for gene delivery include low cytotoxicity, high water solubility, stability, biocompatibility, and capability to bind target molecules. ${ }^{6}$

Dendrimers are macromolecules with a highly geometrically symmetric structure and a large number of positive- or negative-charged modifiable functional groups 
on the surface. Their properties of water-solubility and biocompatibility make dendrimers suitable nanosized gene drug carriers. ${ }^{7}$

Polyamidoamine (PAMAM) dendrimers are positivecharged dendrimers, which have attracted great interest in recent years. They have been successfully developed as gene and drug carriers and imaging agents. ${ }^{6,8-11}$ Their applications in drug delivery helped optimize in vivo distribution of drugs and passive tumor targeting through the EPR effect. ${ }^{4}$ By taking advantage of the modifiable amino groups on the surface, researchers have conjugated different ligands, such as biotinavidin, ${ }^{12}$ lysine, ${ }^{13,14}$ and $\mathrm{PEG}^{15-17}$ onto PAMAM surfaces to enhance transfection efficiency in vivo and in vitro. Recent studies reveal that PAMAM dendrimers activated by timecontrolled heat treatment can make dendriplexes more flexible and exhibit higher transfection efficiency, ${ }^{18,19}$ even though such activation may cause the sacrifice of superficial charges by losing some branches of the dendrimer. The effect of such activation in ligand modification and cytotoxicity remains unclear.

The epidermal growth factor receptor (EGFR) is overexpressed in many human tumors, eg, cancer of the breast, lung, colon, and head and neck, ${ }^{20}$ As targeting ligands, EGF and EGFR antibodies have already been used to increase tumor site enrichment and cell uptake of drug carriers. ${ }^{21,22}$ Most of these attempts are chemistry approaches developed to conjugate EGF onto dendrimer surfaces, which demonstrated significantly increased transfection efficiency to EGFRpositive tumor cells. ${ }^{23}$ However, the conjugation protocol is complicated and might possibly damage the biological activity of EGF. Furthermore, the EGFR-signaling pathway is involved in the regulation of cell proliferation, survival, differentiation and mobility, ${ }^{24}$ and superagonist activity of the EGF-modified dendrimer has been observed. ${ }^{25}$ Therefore, EGF-modified materials should be carefully considered for their potential agonist effect.

Molecule self-assembly is an effective nanosized complex preparation approach. ${ }^{26}$ It is faster than conjugation methods and ensures stability of the complex and biological activity of the biomolecules within. In addition, this approach provides the advantage of flexibility in composition and the capability of inexpensive and large-scale production of the complexes.

In this study, we derived nanosized, self-assembled EGF dendriplexes by binding negatively charged EGF to activated PAMAM dendrimer DNA complexes via electrostatic absorption. These complexes are expected to have comparatively high transfection efficiency to EGFR-positive cells as the conjugation approach and a low transfection rate to EGFR negative cells in order to achieve target selectivity in gene delivery. We investigated properties of the complexes in several experiments, including examining transfection efficiency in MCF-7/EGFR, HEK 293T, and MDA-MB-231 cell line in vitro by fluorescence microimaging and flow cytometry analysis, testing cytotoxicity and possible agonist effect by MTT assay, monitoring biodistribution by in vivo imaging technique, and evaluating transfection efficiency in EGFR-positive tumor in vivo.

\section{Methods and materials Materials}

PAMAM dendrimers G5 were a gift from Prof Yunjun Luo of the Beijing Institute of Technology (Beijing, China). The activated dendrimers were obtained by heating PAMAM G5 water solutions $(1 \mathrm{mg} / \mathrm{mL})$ in a water bath at $40^{\circ} \mathrm{C}$ for 12 hours. ${ }^{18}$ The plasmids pEGFP-N3 encoding EGFP (Clontech, Mountain View, CA), pRL-CMV encoding Renilla luciferase (Promega, Beijing, China) and pFLAG-CMV2 empty vector (Sigma-Aldrich, St Louis, MO) were used in the transfections studies. Kodak X-Sight 670 Large Stokes Shift Dye was purchased from Carestream (Carestream Health, Rochester, NY). All other reagents were obtained from the Biodee Reagent Company (Beijing, China).

\section{Cell culture}

MDA-MB-231 and HEK 293T were obtained from the China Center for Type Culture Collection (Wuhan, China). MCF-7/ EGFR was established according to the reported procedures. ${ }^{27}$ MDA-MB-231/luc cell line was a gift from Prof Chen of Tsinghua University (Beijing, China). MCF-7/EGFR and HEK $293 \mathrm{~T}$ were maintained at $37^{\circ} \mathrm{C}$ under $5 \% \mathrm{CO}_{2}$ in Dulbecco's modified Eagle's medium (Gibco, Beijing, China), and MDA-MB-231 were cultured at $37^{\circ} \mathrm{C}$ in normal atmosphere in Leibovitz's L-15 medium (Gibco). Both mediums were supplemented with $10 \%(\mathrm{v} / \mathrm{v})$ heat-inactivated fetal bovine serum (FBS), penicillin (100 units/mL), and streptomycin $(100 \mu \mathrm{g} / \mathrm{mL})($ Gibco).

\section{Plasmid preparation}

Plasmid was amplified in Escherichia coli (strain DH5 $\alpha$ ), and then isolated and purified using an endotoxin-free plasmid Giga Kit (Tiangen, Beijing, China), according to the manufacturer's instructions. The concentration and purity of the plasmid were assessed using the UV-6300 Spectrophotometer (Mapada, Shanghai, China) at $260 \mathrm{~nm}$ and $280 \mathrm{~nm}$. Plasmid integrity was confirmed by $0.8 \%$ agarose gel electrophoresis and stored at $-20^{\circ} \mathrm{C}$ until further use. 


\section{Preparation of dendriplexes and EGF-dendriplexes}

Dendriplexes (activate or nonactivated)were formed by incubating the two components together in PBS (150 mM NaCl, $1.9 \mathrm{mM} \mathrm{NaH}_{2} \mathrm{PO}_{4}, 8.1 \mathrm{mM} \mathrm{NaH} \mathrm{PO}_{4}$, $\mathrm{pH} 7.4$ ) for 15 minutes at $37^{\circ} \mathrm{C}$. Charge ratios (N/P) were calculated based on the number of terminal amine groups on a PAMAM dendrimer and the number of phosphate groups in the plasmid DNA for both activated and nonactivated dendrimers. In this study, the weight ratio of PAMAM/DNA is 17 when the charge ratio (N/P) is 20 . EGF-dendriplexes were prepared by adding EGF to the preformed dendriplexes. The required amount of EGF was added to the preformed dendriplexes and vortexed in PBS. EGF-dendriplexes were then formed after incubation for another 15 minutes at $37^{\circ} \mathrm{C}$. The weight ratio of EGF and plasmid DNA was from 0.2 to 20 .

\section{Characterization}

Dendriplexes prepared at different EGF/DNA weight ratios $(0,0.2,2$, and 20,4 groups in total). Zeta potential and size (hydrodynamic diameter) were measured by using the Nano-ZS90 Zetasizer (Malvern Instruments, Malvern, UK). Dynamic light scattering was used for size measurements. All measurements were carried out on the dendriplexes with $5 \mu \mathrm{g} / \mathrm{mL}$ plasmid DNA in PBS at $\mathrm{pH}$ 7.4.

\section{Gel retardation assay}

Differently activated EGF-dendriplexes were prepared by incubating in PBS at room temperature for 30 minutes. Each sample was analyzed by electrophoresis on a $0.8 \%$ agarose containing $\mathrm{EB}(0.5 \mu \mathrm{g} / \mathrm{mL})$ at $80 \mathrm{~V}$ for 1 hour. The location of the DNA was identified under UV irradiation.

\section{DNA condensation}

DNA condensation was monitored by ethidium bromide (EB) interaction assay. ${ }^{28}$ Briefly, $1 \mu \mathrm{L}$ EB solution $(0.5 \mathrm{mg} / \mathrm{mL})$ was added to $100 \mu \mathrm{L}$ blank solution (PBS,

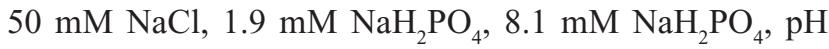
7.4), $100 \mu \mathrm{L}$ activated or nonactivated dendriplexes, and EGF-dendriplexes solutions. After incubation for 2 minutes at room temperature, the fluorescence was measured and analyzed by using the TECAN Safire2 Multimode Reader (TECAN, Shanghai, China) with excitation and emission wavelength at $260 \mathrm{~nm}$ and $600 \mathrm{~nm}$, respectively. Results were expressed as relative fluorescence (\%) to DNA control and were corrected for background fluorescence of free EB in solution.

\section{Stability of activated EGF-dendriplexes}

Six mixtures, ie, naked plasmid DNA, nonactivated dendriplexes, nonmodified activated dendriplexes, and activated EGF-dendriplexes (at different EGF/DNA weight ratios, 0.2-20), were prepared with a fixed final DNA concentration of $50 \mu \mathrm{g} / \mathrm{mL}$ and a total volume of $100 \mu \mathrm{L}$. Each mixture was incubated with DNaseI (5 U/ $\mu \mathrm{g}$ of plasmid DNA) at $37^{\circ} \mathrm{C}$ for 1 hour. $3 \mu \mathrm{L}$ of EDTA $(0.5 \mathrm{M})$ solution was added to stop the DNA degradation, and SDS was added to $1 \%$ final concentration in order to disassemble the complexes. After that, all the samples were incubated for 1 hour and analyzed by $0.8 \%$ agarose gel electrophoresis to evaluate the integrity of DNA in the dendriplexes.

\section{Transfection in vitro}

Activated and nonactivated EGF-dendriplexes were prepared by mixing $1 \mu \mathrm{g}$ of pEGFP-N3 at N/P of 20 at different EGF/ DNA weight ratios $(0.2-20)$.

Cells were seeded at a density of $10^{5}$ cells per well in a 24 well culture plate and grown for 24 hours. Next, the medium was removed and washed twice with PBS. Subsequently, $50 \mu \mathrm{L}$ of the transfection complexes (containing $1 \mu \mathrm{g}$ of plasmid DNA) and $0.2 \mathrm{~mL}$ opti-MEM (Gibco, Beijing, China) were added to each well successively and incubated for 4 hours at $37^{\circ} \mathrm{C}$ with the growth medium for another 48 hours. Qualitative assessment of pEGFP expressed in transfected cells was done by observing the cells under a fluorescent microscope (Leica DM-IL; Leica Microsystems, Brannockburn, IL).

\section{Determination of transfection efficiency}

After transfection, cells were detached by trypsin (Gibco). Cell suspensions were then transferred to microtubes (BD, Franklin Lakes, NJ) and fixed by $0.2 \mathrm{mM}$ EDTA. The percentage of cells transfected was quantitatively assessed at 48 hours after transfection by flow cytometry by fluorescein isothiocyanate (emission 530/30 bandpass) filter using fluorescence-activated cell sorting (FACS) machine (FACSAria system, argon ion laser $488 \mathrm{~nm}$; BD). For each sample, 10,000 events were collected and fluorescence was detected. Transfection efficiency was calculated based on the percentage of the cells that expressed pEGFP (positive cells) in the total number of cells.

\section{MTT assay for studies of cytotoxicity and agonist effect}

MCF-7/EGFR cells were seeded at a density of $1 \times 10^{4}$ cells per well in $200 \mu \mathrm{L}$ medium in a 96-well plate. After 
an overnight culture, cells were treated with activated or nonactivated dendriplexes or EGF-dendriplexes prepared at N/P ratio 20 . For the cytotoxicity study, all dendriplexes contained $1 \mu \mathrm{g}$ of empty pFLAG-CMV2 plasmid DNA, and the MTT assay was administered 4 hours post-transfection. For the agonist effect study, all dendriplexes contained $1 \mu \mathrm{g}$ of empty pFLAG-CMV2 plasmid DNA, and the MTT assay was administered 4 hours and 72 hours post-transfection.

After the above required treatment, $20 \mu \mathrm{L}$ of MTT ( $5 \mathrm{mg} / \mathrm{mL}$ ) was added, and the cells were incubated for approximately 4 hours. The growth medium was removed and $200 \mu \mathrm{L}$ of dimethyl sulfoxide was added to dissolve the MTT crystals. The optical density was read using the Safire2 Multimode microplate reader (TECAN) with $570 \mathrm{~nm}$ as the excitation wavelength and $630 \mathrm{~nm}$ as the background. Viability of cells exposed to dendriplexes was expressed as a percentage of the viability of cells grown in the absence of dendriplexes.

\section{Biodistribution in vivo}

For the circulation kinetics and biodistribution of activated EGF dendriplexes, activated PAMAM dendrimers were labeled with Kodak X-Sight 670 Large Stokes Shift Dye (LSS dye; Kodak, Rochester, NY) by the covalent combination between primary amino groups of dendrimers and the functional ester group of LSS dye. The labeling reaction and purification of LSS dye-labeled PAMAM dendrimers were according to the manufacturer's instructions.

Female BALB/c nude mice (4 weeks of age) were purchased from the Experimental Animal Center (Tsinghua University, Beijing, China). Animal use was approved by Committee on Animal Research in Tsinghua University. Tumors were introduced in the armpits of the nude mice by inoculation with $1 \times 10^{6}$ of luciferase gene labeled MDAMB-231/luc cells. Palpable subcutaneous tumors developed over a period of approximately 7 days. Distribution and metabolism was assessed by injecting LSS dye-labeled activated EGF dendriplexes (EGF/DNA weight ratio of 2, $\mathrm{N} / \mathrm{P}$ ratio of 20) and LSS dye-labeled nonmodified activated dendriplexes (N/P ratio of 20) into tumor-bearing mice in groups of six via the tail vein in a total volume of $200 \mu \mathrm{L}$ (containing $30 \mathrm{ug}$ plasmid DNA per mouse).

The Kodak in vivo imaging system FX-Pro was used for image acquisition in vivo, and images were analyzed with the Carestream MI SE 5.0.7.23 software package. For tumor bioluminescence imaging analysis, D-luciferin was used. $150 \mathrm{mg} / \mathrm{kg}$ D-luciferin was injected intraperitoneally. Five minutes later, the mice were anesthetized with $2.5 \%(\mathrm{v} / \mathrm{v})$ isoflurane and placed in the dark chamber of the Kodak in vivo imaging system FX-Pro for light acquisition.

\section{Transfection in vivo}

Female BALB/c mice (4 weeks of age) were purchased from the Experimental Animal Center (Tsinghua University). All animals were approved by Committee on Animal Research in Tsinghua University. Tumors were introduced in the armpits of the mice by inoculation with $1 \times 10^{6}$ of MCF-7/EGFR cells (which exhibits the biggest enhancement and the highest transfection efficiency, as they did in activated EGF-dendriplexes in our in vitro study). Gene expression in vivo was assessed by injecting activated EGFdendriplexes containing pRL-CMV, at EGF/DNA weight ratio of 0 (nonmodified), 2, 20 (both at an N/P ratio of 20) into 4-week-old individual mice in groups of six via the tail vein in a total volume of $200 \mu \mathrm{L}$. Tumor tissues were collected 24 hours post-intravenous injection, washed twice with cold saline, and homogenized with lysis buffer (Promega) using the homogenizer (DY89-II; Huadr, Beijing, China) and centrifuged at $12,000 \mathrm{~g}$ for 5 minutes at $4^{\circ} \mathrm{C}$. Levels of Renilla luciferase activity in the transfected cells were assayed using the Renilla Luciferase Assay Kit (Promega), according to the manufacturer's instructions.

\section{Statistical analysis}

Statistical analysis was performed using two-way analysis of variance (Origin software, version 7.5; Origin Lab, Northampton, MA). $P<0.05$ was considered statistically significant.

\section{Results and discussion Characterization, formation, and stability of activated EGF-dendriplexes}

At the charge ratio (N/P) of 20, we investigated the impact of EGF dosage on dendriplex size (hydrodynamic diameter) and zeta potential. Dendriplexes formed by activated dendrimer are referred to as activated dendriplexes, and dendriplexes formed by nonactivated dendrimer are referred to as nonactivated dendriplexes hereafter. As shown in Table 1, as the dosage of EGF increases, the zeta potential declines significantly, whereas dendriplex size remains steady, except when the EGF/DNA ratio is elevated to 20. Under this condition, EGF-dendriplex size rises dramatically to over $900 \mathrm{~nm}$ and $1000 \mathrm{~nm}$ in the activated state and nonactivated state, respectively. The standard deviation of dendriplex size also increases, indicating that the zeta-potential is too low to form complexes with a steady structure. 
Table I Size and zeta-potential of activated and nonactivated EGF-dendriplexes

\begin{tabular}{|c|c|c|c|c|}
\hline \multirow[t]{2}{*}{ Weight ratio of EGF/DNA } & \multicolumn{2}{|l|}{ Size $(\mathrm{nm})$} & \multicolumn{2}{|c|}{ Zeta-potential (mV) } \\
\hline & Activated & Nonactivated & Activated & Nonactivated \\
\hline 0 & $358.7 \pm 6.9$ & $365.9 \pm 4.5$ & $16.2 \pm 0.2$ & $18.3 \pm 0.3$ \\
\hline 0.2 & $369.5 \pm 8.4$ & $354.3 \pm 10.4$ & $11.7 \pm 0.1$ & $14.2 \pm 0.2$ \\
\hline 2 & $366.2 \pm 3.3$ & $370.4 \pm 5.2$ & $6.8 \pm 0.1$ & $7.9 \pm 0.1$ \\
\hline 20 & $983.8 \pm 51.5$ & $1012.7 \pm 70.5$ & $2.4 \pm 0.8$ & $3.3 \pm 0.5$ \\
\hline
\end{tabular}

Note: $\mathrm{n}=4$ and 6 for size and zeta-potential measurements, respectively.

Dendrimers can block access to the DNA of other molecules once they form dendriplexes, thus prohibiting the DNA from EB dyeing or digestion. To rule out the possibility that heating activation and EGF modification may destabilize the DNA-dendrimer interaction, we examined the retardation in the migration of the plasmid DNA during agarose gel electrophoresis, performed an ethidium bromide (EB) dye exclusion assay, and investigated the stability of the complexes using a DNaseI digestion test.

Agarose gel electrophoresis (Figure 1A) shows that at $\mathrm{N} / \mathrm{P}=20$, the mobility of DNA in EGF-dendriplexes with variant EGF dosage is indistinctively retarded. In addition, the EB dye exclusion assay (Figure 1B) demonstrates that there are no significant differences in the relative fluorescence units among nonactivated dendriplexes, activated dendriplexes, and activated EGF-dendriplexes at different weight ratios of $\mathrm{EGF} / \mathrm{DNA}$ when the charge ratio $\mathrm{N} / \mathrm{P}=20$. Results of the DNaseI digestion test (Figure 1C) show that both activated and nonactivated dendriplexes can protect DNA from being digested, regardless of whether modified by EGF or not. These results suggest that dendriplexes formed by activated dendrimers are as stable as those formed by nonactivated ones in terms of DNA condensation, and selfassembled EGF modification on such dendriplexes has no effect on their stability.

In conjugation methods described in previous studies, molecules are modified onto PAMAM dendrimers before PAMAM-DNA complex formation. However, studies on the influences on PAMAM dendrimer-DNA interaction are limited..$^{15}$ In theory, such approaches may affect interactions between dendrimers and DNA molecules, particularly for negatively charged proteins such as EGF, which increase steric hindrance and electrostatic repulsion against DNA. In self-assembly approaches, on the other hand, modification is administered after formation of PAMAM-DNA complexes, thus avoiding destabilization of the complexes by altered interactions between dendrimers and DNA. Our EB dyeing results provide evidence that EGF modification through self-assembly indeed does not affect the dendrimer-DNA interaction.

\section{EGF enhances the transfection efficiency of dendriplexes in human breast cancer cell line MCF-7/EGFR}

At a fixed concentration of $1 \mu \mathrm{g}$ DNA in dendriplex form per $200 \mu \mathrm{L}$ transfection complexes, we investigated the effect of different weight ratios of EGF/DNA on the transfection efficiency of activated and nonactivated dendriplexes in MCF-7/ EGFR, a human breast cancer cell line stably transfected with full-length EGFR cDNA and over-expressing EGFR. The result (Figure 2) shows that the transfection efficiency of both dendriplexes is positively correlated with EGF/DNA weight ratio. However, at extremely high EGF/DNA weight ratios $(\mathrm{EGF} / \mathrm{DNA}=20)$, transfection efficiency decreases.

In theory, dendriplex binds to cell surface through electrostatic interaction. ${ }^{29}$ Its cellular uptake occurs by clathrin-mediated endocytosis, and its transfection efficiency becomes higher with increasing zeta-potential..$^{30}$ As supported by results in the previous section, when EGF/DNA weight ratio is increased, zeta-potential of EGF-dendriplexes drops, whereas transfection efficiency rises. The inconsistency of zeta-potential and transfection efficiency suggests that other factors are responsible for altered transfection efficiency in this experiment. The fact that activated EGFR also goes through clathrin-mediated endocytosis, as shown by previous studies, ${ }^{24}$ provides a clue. Two possible causes can lead to higher transfection efficiency: (1) EGFR signal pathway activation outweighs lowered zeta potential, promoting clathrin-mediated endocytosis; and (2) EGF ligand-receptor interaction enhances local concentration of EGF-dendriplexes, enabling more contact of EGF-dendriplexes with the cell, thus increasing electrostatic interaction. In addition, as the surface charge of the activated dendrimers is reduced, the structure of activated dendriplexes becomes more stretched out and flexible compared to the condensed nonactivated ones. ${ }^{18}$ As a result, activated dendriplexes have more chances to contact with and be taken up by the cell in spite of lowered zeta-potential. 


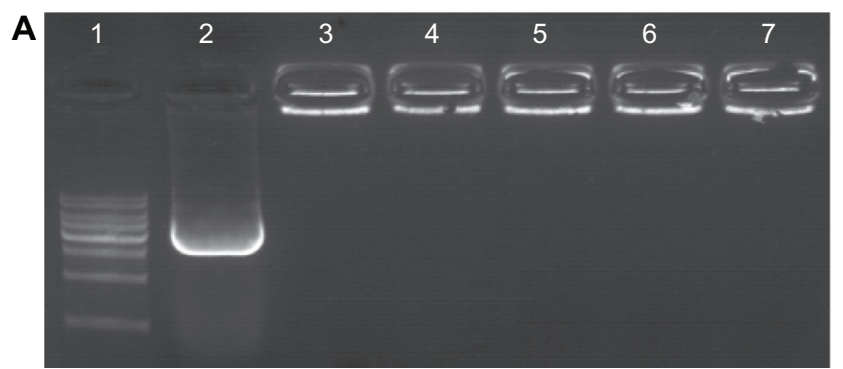

B
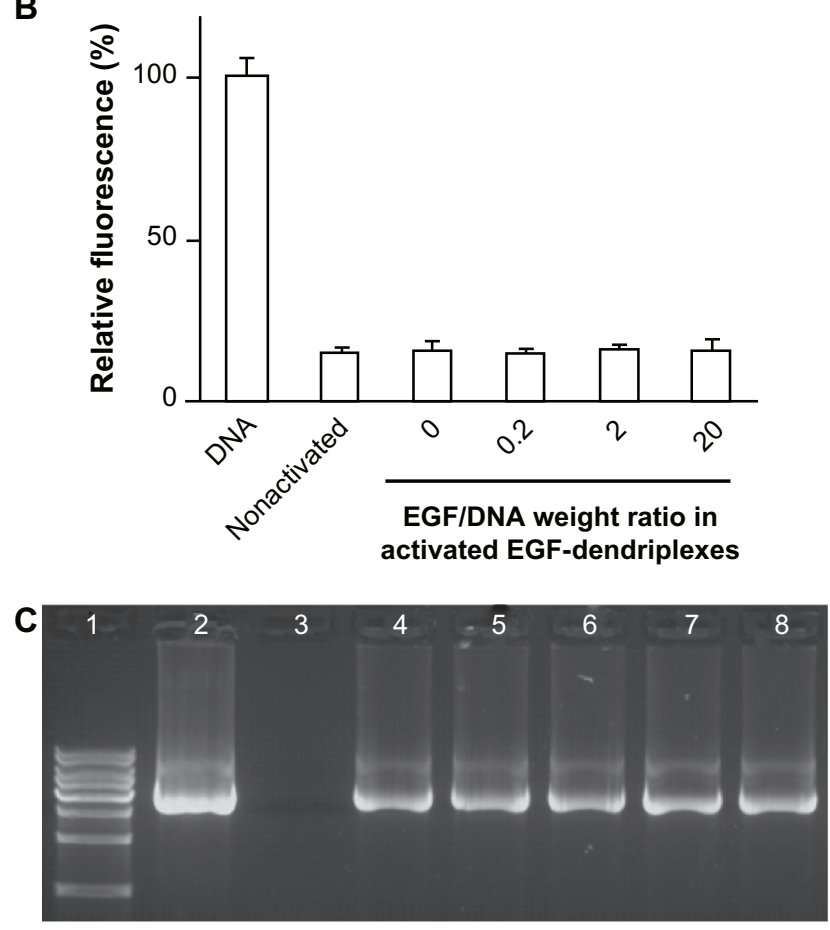

Figure I Formation and stability analysis of activated EGF-dendriplexes. (A) Agarose gel retardation assay of activated EGF-dendriplexes at different EGF/ DNA weight ratio. Lane I: I kb DNA ladder; lane 2: plasmid DNA; lane 3: nonactivated dendriplexes at charge ratio (N/P) of 20; lane 4: activated dendriplexes at charge ratio (N/P) of 20; Lane 5-7: activated EGF-dendriplexes at charge ratio (N/P) of 20 and EGF/DNA weight ratio of 0.2 , 2, and 20 respectively. (B) DNA condensation assay of nonactivated dendriplexes, activated dendriplexes, and activated EGFdendriplexes at EGF/DNA weight ratio of 0.2, 2, and 20. Mean \pm standard deviation $(n=6)$ of relative viability is shown. (C) DNasel digestion test of dendriplexes. Lane I: I kb DNA ladder; lane 2: plasmid DNA without digestion; lane 3-8: DNA in different form post digestion and disassembly of complexes. Lane 3: free plasmid DNA; lane 4: DNA in nonactivated dendriplexes at charge ratio (N/P) of 20; lane 5 : DNA in activated dendriplexes at charge ratio (N/P) of 20; Lane 6-8: DNA in activated EGF-dendriplexes at charge ratio (N/P) of 20 and EGF/DNA weight ratio of $0.2,2$, and 20 , respectively.

Note: DNA indicates naked plasmid DNA; nonactivated indicates nonactivated dendriplexes.

Apart from cellular uptake, DNA release intracellular is another key step in transfection. As mentioned above, it is highly possible that EGF dendriplexes taken through clathrin-mediated endocytosis induced by either EGFR pathway activation or electrostatic interaction will ultimately appear in lysosome, ${ }^{24}$ where the proton sponge effect of dendrimers damages the lysosome structure under low $\mathrm{pH}$ and releases the DNA within.${ }^{31}$ In this process, poorly charged dendrimers in the less condensed activated dendriplexes come off more easily from DNA than the nonactivated ones, thus releasing more DNA into the cytoplasm, resulting in higher transfection efficiency.

\section{Transfection efficiency in EGFR negative cell line HEK 293T and another EGFR- positive human breast cancer cell line MDA-MB-23 I}

In addition to experimentation on the MCF-7/EGFR cell line, we tested transfection efficiency in EGF-dendriplexes in other cell lines as a preliminary in vitro investigation of targeting effect study in vivo. We chose the EGFR negative Human Embryonic Kidney 293T cell line, low EGFRexpressing ( HEK 293T) as an example of EGFR-negative body tissues, and EGFR overexpressing MDA-MB-231 as an example of an EGFR-positive tumor cell line. ${ }^{32}$ We transfected activated dendriplexes, activated EGF-dendriplexes, nonactivated dendriplexes, and nonactivated EGF-dendriplexes in these cell lines, and compared the transfection efficiency. As shown in Figure 3, in EGFR negative HEK 293T, EGF dendriplex has significantly lower transfection efficiency than unmodified dendriplex, whereas in EGFR overexpressing MDA-MB-231, EGF modification notably increases transfection efficiency.

This contrast is likely due to the combination of more greatly reduced zeta-potential in EGF dendriplex than in nonmodified dendriplex as well as the targeting effect of EGF molecules. In EGFR-negative HEK 293T, the absence of targeting receptor and lowered zeta-potential result in lowered transfection efficiency. In EGFR overexpressing MDAMB-231, the targeting effect overcomes the disadvantage in zeta-potential, and a net increase in transfection efficiency is observed, as it is in the assay of the MCF-7/EGFR cell line.

\section{Cytotoxicity of activated and nonactivated EGF-dendriplexes}

We investigated EGF's effect on the toxicity of activated and nonactivated EGF dendriplexes by MTT assay, measured by relative viability. As shown in Figure 4, nonactivated dendriplexes exhibit generally higher toxicity than the activated group, but the gap is insignificant when EGF/ DNA weight ratio is as high as $20(P>0.05)$. Cellular toxicity descends as the amount of EGF increases in both activated and nonactivated groups. In the activated dendriplexes group, EGF dendriplexes have significantly lower toxicity than the nonmodified dendriplexes $(\mathrm{EGF} /$ DNA $=0$ ), but increasing amounts of EGF amount do not reduce toxicity further. 
A

0
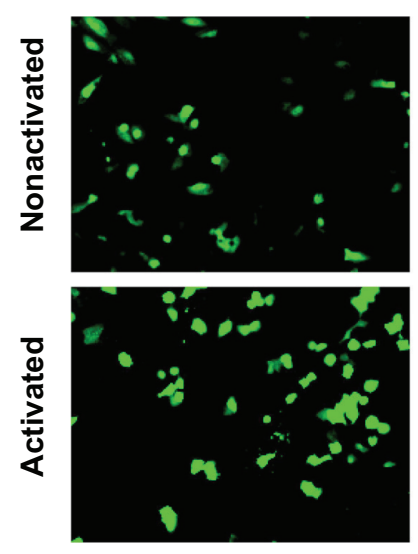

EGF/DNA weight ratio

0.2
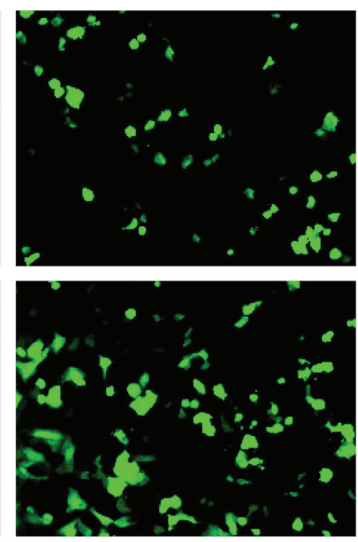

2
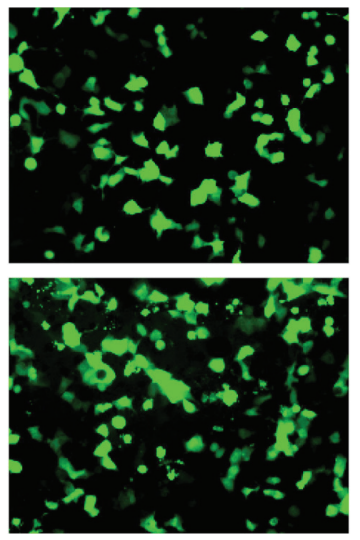

20
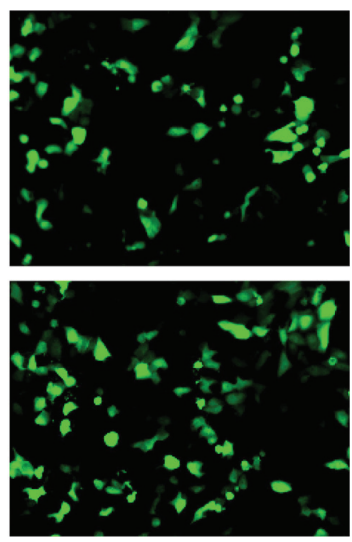

B

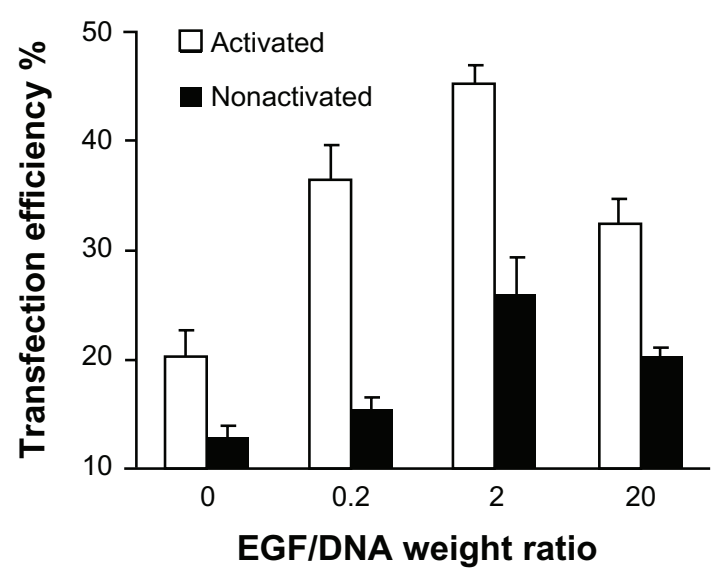

Figure 2 Expression of enhanced green fluorescent protein (EGFP) in MCF-7/EGFR cells transfected with pEGFP-N3. (A) Scanned through a fluorescence microscope; (B) tested by flow cytometry analysis.

Note: Mean \pm standard deviation $(n=3)$ of transfection efficiency is shown.

The reduction of short-term (4 hour) cytotoxicity can be attributed to the cellular uptake channel of dendriplexes. Clathrin-mediated endocytosis triggered by electrostatic interaction, as the only possible way for nonmodified dendriplexes to enter the cell, may damage the lipid bilayer structure of cell membrane and lead to cell death, ${ }^{6}$ whereas EGF-dendriplexes can be taken up by the cell through EGFR signal pathway triggered endocytosis, a normal physiological process, which therefore causes less damage to the cell.

\section{The agonist effect of activated EGF- dendriplexes on EGFR-positive human breast cancer cell line}

In the experiments reported previously, the overall targeted transfection efficiency and cytotoxicity were considered satisfactory. Before its application in cancer gene therapy, we must also confirm that activated EGF dendriplexes have low or no agonist effect on cell proliferation. Here we present the result of an MTT assay to test the effect of EGF-containing transfection complexes on cell proliferation of MCF-7/ EGFR cell line.

As shown in Figure 5, after 4 hours of transfection, the two groups of cells with added activated EGF dendriplexes (including $2 \mu \mathrm{g}$ and $20 \mu \mathrm{g}$ EGF in complexes, respectively) displayed no significant viability differences from the control cells $(97.7 \% \pm 3.4 \%, 97.7 \% \pm 10.3 \% ; P>0.05)$, indicating that under the selected EGF concentration, cell proliferation is not promoted significantly by EGF. The dissociative EGF groups, working as positive control, contain the same amounts of EGF per $200 \mu \mathrm{L}$ medium as the corresponding EGF dendriplex groups. Dissociative EGF at $2 \mu \mathrm{g}$ per $200 \mu \mathrm{L}$ medium does not stimulate cell proliferation significantly $(108.7 \% \pm 7.3 \% ; P>0.05)$. However, at $20 \mu \mathrm{g}$ EGF per $200 \mu \mathrm{L}$ medium concentration (equivalent to EGF/ DNA $=20$ dendriplex group), it promotes cell proliferation at a significant level $(156.0 \% \pm 13.1 \%)$.

After 3 days of incubation from transfection termination, the groupwise viability differences are fewer com- 
A
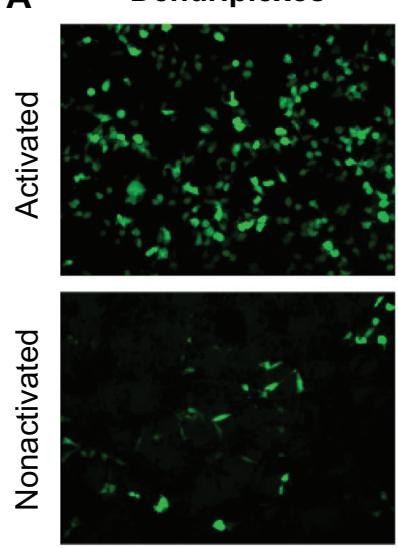

B
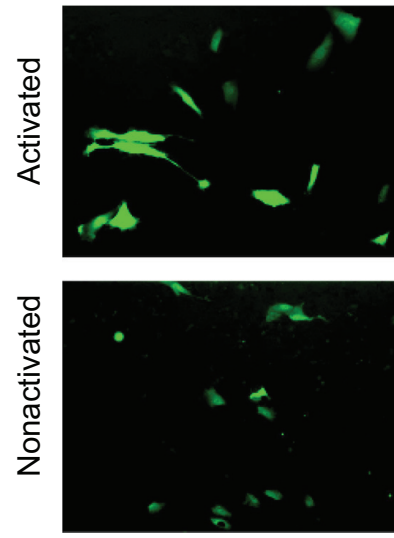

Figure 3 Expression of enhanced green fluorescent protein (EGFP) in different cell lines transfected with pEGFP-N3 scan through a fluorescence microscope. (A) EGFR-negative HEK 293T cells; (B) EGFR-positive human breast cancer MDAMB-23I cells.

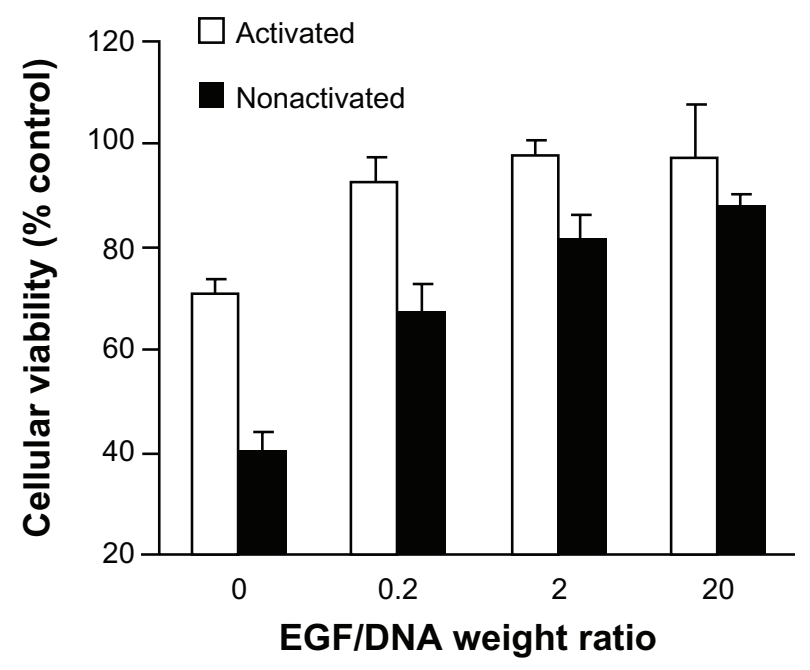

Figure 4 The viability of MCF-7/EGFR cells 4 hours after adding activated or nonactivated EGF-dendriplexes $(N / P=20)$ with different weight ratio of EGF/DNA. Note: Mean \pm standard deviation $(n=6)$ of relative viability is shown. Viability is calculated from average OD 570 -fold change relative to control culture.

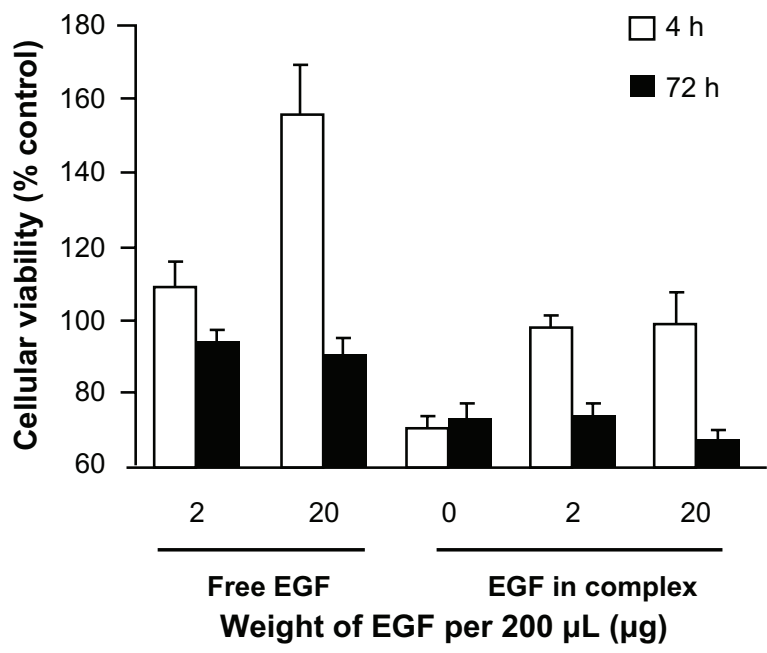

Figure 5 The viability of MCF-7/EGFR cells administrated with EGF-dendriplexes or equivalent amount of free EGF.

Notes: Viability is tested immediately after 4 hours of transfection, and 3 days under incubation after transfection termination. Relative viability to control cells is shown. Mean \pm standard deviation $(n=6)$ of relative viability is shown.

pared to those immediately after transfection termination. Cells treated with different EGF weight show indistinctive $(P>0.05)$ viability for dissociative EGF groups and EGF in complexes groups, respectively.

When the targeted delivery of drugs or genes using EGF is desired, the EGFR signaling pathway is activated, which stimulates cell proliferation. ${ }^{24}$ Previous studies showed that EGF dendrimer conjugates are stronger superagonists than free EGF. ${ }^{25}$ However, in our results, EGF dendriplexes did not display stronger cell growth-stimulating properties than free EGFs. There are two possible causes. First, each EGF-dendriplex is capped with multiple EGF molecules, so that the number of EGF molecules accessible to act as agonist is greatly reduced, whereas the aforementioned EGF-dendrimer conjugates used for chemical drug carriers or imaging are all accessible. Thus, with equivalent EGF concentration, EGF dendriplexes show lower cell growth-stimulating properties. The second possible reason that EGF-dendriplex are not as strong in agonizing cell proliferation as expected, is that in the EGFR signaling pathway, the EGF/EGFR complex needs to translocate into the nucleus to trigger cell proliferation. ${ }^{24}$ Different from EGF dendrimer conjugates, which are similar in size to dendriplex $\left(\sim 10^{0} \mathrm{~nm}\right) .{ }^{1,23,25}$ the larger-sized EGF dendriplexes $\left(\sim 10^{2} \mathrm{~nm}\right)$ cannot pass directly through nuclear pores $\left(\sim 10^{1} \mathrm{~nm}\right)$, so that the nuclear import process of EGF/EGFR complexes in EGFR-signaling pathway is blocked or slowed down. This feature of activated EGF-dendriplexes may prevent unexpected interactions between dendrimers and endonuclear 
components, which may cause dysfunctional deactivation of the EGFR-signaling pathway. ${ }^{25}$

\section{The biodistribution of activated EGF-dendriplexes in vivo}

We performed near-infrared (NIR) fluorescence imaging to investigate the biodistribution of EGF-modified and nonmodified activated dendriplexes. Dendriplexes formed by LSS dye-labeled activated dendrimer was observed by noninvasive NIR fluorescence optical imaging technology in vivo. As shown in Figure 6, within the selected scale range of fluorescence counts, stronger signals and clearer boundar-

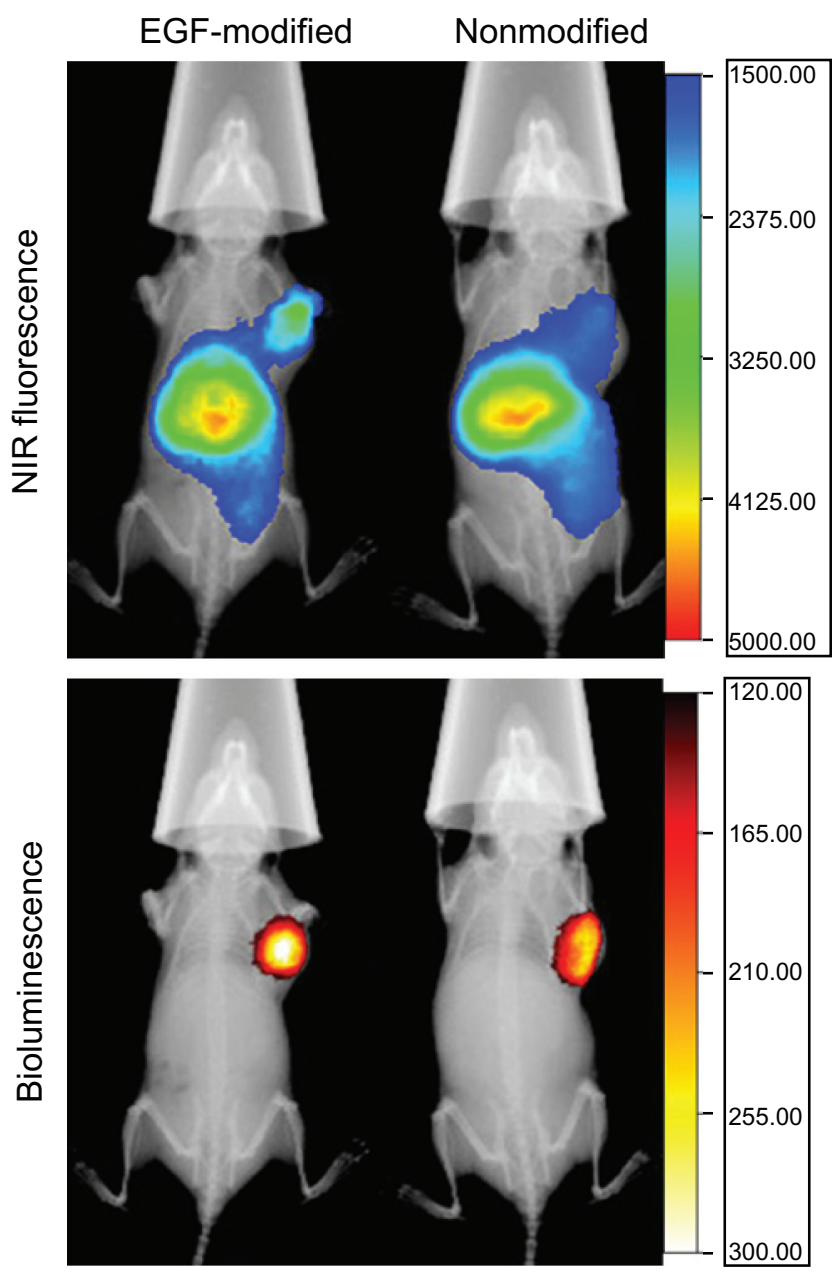

Figure 6 X-ray, in vivo NIR fluorescence and bioluminescence overlay images of tumor-bearing mice 2 hours after being treated with activated dendriplexes and activated EGF-dendriplexes.

Notes: The location of MDA-MB-23I-luc tumor was determined with bioluminescence by injecting $150 \mathrm{mg} / \mathrm{kg}$ D-luciferin. The optical imaging was obtained using Kodak multimodal imaging system FX-Pro equipped with an excitation bandpass filter at $690 \mathrm{~nm}$ and an emission at $790 \mathrm{~nm}$. The calibration bar shows the fold increase in fluorescence counts from the minimum fluorescence counts EGF-modified indicates activated EGF-dendriplexes; nonmodified indicates activated dendriplexes. Abbreviations: NIR, near-infrared fluorescence. ies of NIR fluorescence were observed 2 hours post-treatment at tumor site in the group treated with activated EGF dendriplexes. High signals were observed at the abdomens of mice from both groups. The location of MDA-MB-231/ luc tumor was determined with bioluminescence.

Nanosized complexes formed by macromolecules together with DNA plasmids are able to target tumor sites passively for their EPR effect, which is mainly caused by the abundant newly formed abnormal tumor vessels and the lack of effective lymphatic drainage in the tumor site.,33 That is the reason weak signals and fuzzy boundary of NIR fluorescence were observed at the tumor site in the group treated with nonmodified activated dendriplexes. Regardless of whether dendriplexes are modified by EGF or not, NIR fluorescence signals are found at presumable liver regions, indicating that PAMAM dendriplexes might metabolize in this organ.

\section{Transfection in vivo}

To evaluate the possible targeting gene delivery in vivo, we tested exogenous Renilla luciferase expression levels in MCF-7/EGFR tumor tissues. Renilla luciferase was transfected by intravenous administrated nonmodified and EGF-modified activated dendriplexes of different EGF/DNA weight ratios $(\mathrm{EGF} / \mathrm{DNA}=2,20)$. Our result shows that this self-assembled EGF modification can significantly enhance the expression levels of dendriplex transfected Renilla luciferase in tumor tissue (Figure 7). Unlike the in vitro results, the activated EGF-dendriplex group with weight ratio

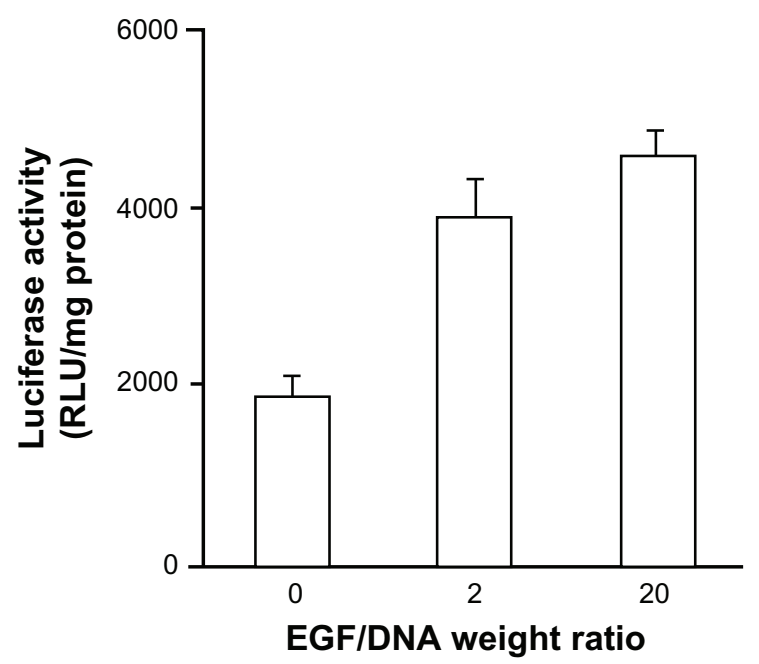

Figure 7 In vivo gene expression in MCF-7/EGFR tumor tissues 24 hours postintravenous injection of nonmodified activated dendriplexes and activated EGFdendriplexes at EGF/DNA weight ratio of 2 and 20.

Note: Mean \pm standard deviation $(n=3)$ of Renilla luciferase activity is shown. 
of EGF/DNA = 20 exhibited higher luciferase expression (4563 $\pm 322 \mathrm{RLU} / \mathrm{mg}$ protein) than the $\mathrm{EGF} / \mathrm{DNA}=2$ group (3865 $\pm 484 \mathrm{RLU} / \mathrm{mg}$ protein) in vivo.

This observation can be explained by competitive binding between preassembled EGF and other serum components. Positively charged dendriplexes can interact with negatively charged molecules in the plasma post-intravenous injection, some of which may even dissociate DNA from the complexes. ${ }^{34}$ The higher the EGF/DNA weight ratio before injection, the fewer vacancies dendriplexes offer for negatively charged molecules to bind, which maintains a more stable structure and a higher targeting specificity to EGFR-positive tumor cells of complexes in vivo.

\section{Conclusion}

In summary, we developed novel nanosized EGF-modified dendriplexes via self-assembly of EGF, DNA, and activated PAMAM dendrimers, and then tested their performances as gene carriers in human cell lines. Our dendriplexes show high transfection efficiency as well as low cytotoxicity and agonist effect in vitro, EGFR-positive tumor targeted biodistribution in vivo, and enhancement of transfection efficiency in tumor tissues in vivo. Our results suggest that as a novel nanosized gene-delivery system, this self-assembled activated EGF dendriplex is a potential candidate for EGFR-targeted cancer therapy.

\section{Acknowledgments}

This work was supported by the National 973 Program (2009CB903300), the Tsinghua-Yue Yuen Medical Research Fund (20240000572), and the Key New Drug Creation and Development Program (2012ZX09103301-31).

\section{Disclosure}

The authors report no conflicts of interest in this work.

\section{References}

1. Gillies ER, Frechet JM. Dendrimers and dendritic polymers in drug delivery. Drug Discov Today. 2005;10(1):35-43.

2. Sampathkumar SG, Yarema KJ. Targeting cancer cells with dendrimers. Chem Biol. 2005;12(1):5-6.

3. Fang J, Nakamura H, Maeda $\mathrm{H}$. The EPR effect: Unique features of tumor blood vessels for drug delivery, factors involved, and limitations and augmentation of the effect. Adv Drug Deliv Rev. 2011;63(3): 136-151.

4. Torchilin V. Tumor delivery of macromolecular drugs based on the EPR effect. Adv Drug Deliv Rev. 2011;63(3):131-135.

5. Petros RA, DeSimone JM. Strategies in the design of nanoparticles for therapeutic applications. Nat Rev Drug Discov. 2010;9(8):615-627.

6. Duncan R, Izzo L. Dendrimer biocompatibility and toxicity. Adv Drug Deliv Rev. 2005;57(15):2215-2237.
7. Lee CC, MacKay JA, Frechet JM, Szoka FC. Designing dendrimers for biological applications. Nat Biotechnol. 2005;23(12):1517-1526.

8. Esfand R, Tomalia DA. Poly(amidoamine) (PAMAM) dendrimers: from biomimicry to drug delivery and biomedical applications. Drug Discov Today. 2001;6(8):427-436.

9. Mamede M, Saga T, Ishimori T, et al. Hepatocyte targeting of 111 In-labeled oligo-DNA with avidin or avidin-dendrimer complex. J Control Release. 2004;95(1):133-141.

10. Svenson S, Tomalia DA. Dendrimers in biomedical applications reflections on the field. Adv Drug Deliv Rev. 2005;57(15):2106-2129.

11. Zhang Y, Sun Y, Xu X, et al. Synthesis, biodistribution, and microsingle photon emission computed tomography (SPECT) imaging study of technetium-99m labeled PEGylated dendrimer poly(amidoamine) (PAMAM)-folic acid conjugates. J Med Chem. 2010; 53(8):3262-3272.

12. Pillai VC, Yesudas R, Shaik IH, et al. Delivery of NADPH-cytochrome $\mathrm{P} 450$ reductase antisense oligos using avidin-biotin approach. Bioconjug Chem. 2010;21(2):203-207.

13. Vlasov GP, Lesina EA, Korol'kov VI, et al. Optimization of transfection properties of DNA-lysine dendrimer complexes. Bioorg Khim. 2005;31(2):167-174.

14. Choi JS, Park JS. Supramolecular self-assembly of poly(ethylene glycol)-block-poly (L-lysine) dendrimer with plasmid DNA. Methods Mol Med. 2001;65:23-33.

15. Froehlich E, Mandeville JS, Weinert CM, Kreplak L, Tajmir-Riahi HA. Bundling and aggregation of DNA by cationic dendrimers. Biomacromolecules. 2011;12(2):511-517.

16. Froehlich E, Mandeville JS, Kreplak L, Tajmir-Riahi HA. Aggregation and particle formation of tRNA by dendrimers. Biomacromolecules. 2011;12(7):2780-2787.

17. Froehlich E, Mandeville JS, Arnold D, Kreplak L, Tajmir-Riahi HA. Effect of PEG and mPEG-anthracene on tRNA aggregation and particle formation. Biomacromolecules. 2012;13(1):282-287.

18. Navarro G, Tros de Ilarduya C. Activated and non-activated PAMAM dendrimers for gene delivery in vitro and in vivo. Nanomedicine. 2009;5(3):287-297.

19. Kuo JH, Liou MJ, Chiu HC. Evaluating the gene-expression profiles of HeLa cancer cells treated with activated and nonactivated poly(amidoamine) dendrimers, and their DNA complexes. Mol Pharm. 2010;7(3):805-814.

20. Kandala PK, Wright SE, Srivastava SK. Blocking EGFR activation suppresses ovarian tumor growth in vitro and in vivo. $J$ Pharmacol Exp Ther. 2012;34(1):24-32.

21. Frederiksen KS, Abrahamsen N, Cristiano RJ, Damstrup L, Poulsen HS. Gene delivery by an epidermal growth factor/DNA polyplex to small cell lung cancer cell lines expressing low levels of epidermal growth factor receptor. Cancer Gene Ther. 2000;7(2):262-268.

22. Chen J, Gamou S, Takayanagi A, Ohtake Y, Ohtsubo M, Shimizu N. Receptor-mediated gene delivery using the Fab fragments of antiepidermal growth factor receptor antibodies: improved immunogene approach. Cancer Gene Ther. 1998;5(6):357-364.

23. Yuan Q, Lee E, Yeudall WA, Yang H. Dendrimer-triglycine-EGF nanoparticles for tumor imaging and targeted nucleic acid and drug delivery. Oral Oncol. 2010;46(9):698-704.

24. Sebastian S, Settleman J, Reshkin SJ, Azzariti A, Bellizzi A, Paradiso A. The complexity of targeting EGFR signalling in cancer: from expression to turnover. Biochim Biophys Acta. 2006;1766(1):120-139.

25. Thomas TP, Shukla R, Kotlyar A, et al. Dendrimer-epidermal growth factor conjugate displays superagonist activity. Biomacromolecules. 2008;9(2):603-609.

26. Zhang Q, Chen S, Zhuo RX, Zhang XZ, Cheng SX. Self-assembled terplexes for targeted gene delivery with improved transfection. Bioconjug Chem. 2010;21(11):2086-2092.

27. Kruger JS, Reddy KB. Distinct mechanisms mediate the initial and sustained phases of cell migration in epidermal growth factor receptoroverexpressing cells. Mol Cancer Res. 2003;1(11):801-809. 
28. Shcharbin D, Pedziwiatr E, Bryszewska M. How to study dendriplexes I: Characterization. J Control Release. 2009;135(3):186-197.

29. Mounkes LC, Zhong W, Cipres-Palacin G, Heath TD, Debs RJ. Proteoglycans mediate cationic liposome-DNA complex-based gene delivery in vitro and in vivo. $J$ Bio Chem. 1998;273(40): 26164-26170.

30. Mislick KA, Baldeschwieler JD. Evidence for the role of proteoglycans in cation-mediated gene transfer. Proc Natl Acad Sci U S A. 1996;93(22):12349-12354.

31. Tang MX, Redemann CT, Szoka FC Jr. In vitro gene delivery by degraded polyamidoamine dendrimers. Bioconjug Chem. 1996;7(6): 703-714.
32. Anderson NG, Ahmad T, Chan K, Dobson R, Bundred NJ. ZD1839 (Iressa), a novel epidermal growth factor receptor (EGFR) tyrosine kinase inhibitor, potently inhibits the growth of EGFR-positive cancer cell lines with or without erbB2 overexpression. Int $J$ Cancer. 2001;94(6):774-782.

33. Duncan R. Polymer conjugates for tumour targeting and intracytoplasmic delivery. The EPR effect as a common gateway? Pharm Sci Technolo Today. 1999;2(11):441-449.

34. Zelphati O, Uyechi LS, Barron LG, Szoka FC Jr. Effect of serum components on the physico-chemical properties of cationic lipid/oligonucleotide complexes and on their interactions with cells. Biochim Biophys Acta. 1998;1390(2):119-133.
International Journal of Nanomedicine

\section{Publish your work in this journal}

The International Journal of Nanomedicine is an international, peerreviewed journal focusing on the application of nanotechnology in diagnostics, therapeutics, and drug delivery systems throughout the biomedical field. This journal is indexed on PubMed Central,

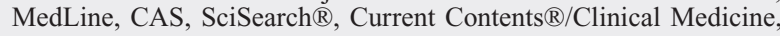

\section{Dovepress}

Journal Citation Reports/Science Edition, EMBase, Scopus and the Elsevier Bibliographic databases. The manuscript management system is completely online and includes a very quick and fair peer-review system, which is all easy to use. Visit http://www.dovepress.com/ testimonials.php to read real quotes from published authors.

Submit your manuscript here: http://www.dovepress.com/international-journal-of-nanomedicine-journal 